\title{
Relative phase stability: The interaction of attentional instruction and frequency on relative phase of bimanual coordination movements
}

\author{
Ehsan ZAREIAN \\ Department of Motor Behavior, Allameh Tabataba'i University, Tehran, Iran. \\ Address Correspondence to E. Zareian, ehsan.zarian@gmail.com
}

\begin{abstract}
The present paper aimed to study the effect of using attentional instruction and increasing the rhythm on the relative phase stability of coordinated out-phase bimanual coordination movements. Using a plotter device, the participants ( $\mathrm{N}=16)$ performed out-phase bimanual coordination pattern $(45,90$, and 135 degree) while focusing on signs of the device (external attention) or their forearm (internal attention) or without attentional instruction with increasing frequency. KP was provided to participants after each attempt. Dependent variables were analyzed by 3 (attention) $\times 3$ (relative phase) $\times 4$ (frequency) analysis of variance with repeated measures on second and third variables. The results revealed that external attention lead to increased stability of relative phase in all three phase patterns. Additionally, increased frequency of motions caused decreased stability of relative in all three patterns. Findings of this study corroborated the constrained action hypothesis and showed that, unlike in-phase and anti-phase attractor patterns and because of the difficulty out-phase patterns, external attention instruction can act contrary to linear changes of control parameter and increase their stability of relative phases.
\end{abstract}

Key words: Attentional instructions, bimanual coordination, frequency, relative phase stability.

\section{INTRODUCTION}

There is increasing evidence suggesting that human motions can be directly influenced by the focus of attention. Attention is part of the data collection process and is divided into two categories of internal focus of attention and external focus of attention (15). Internal focus of attention refers to the use of information by a person which comes back to him/herself (26), while external focus of attention deals with the direction of attention about the effects caused by a movement in the environment, for example, entering or not entering the ball into the basket (24). There is a consensus on the fact that focus on the effects of a motion (external focus of attention) can lead to better results compared with focus on motion pattern (internal focus of attention) $(25,26)$. Constrained action hypothesis claims that the reason for superiority of external focus of attention is facilitation of automatic control of motion. This superiority has been proven in various motor activities such as basketball (27), balance tasks (19), tennis (13), jumping (23), dart throwing (4), and aiming (18). However, some studies showed opposite and paradoxical results. For example, it has been observed that professional golfers benefit more from external focus and beginners mostly get use of internal focus (14). Vance et al. reported that there is no difference between internal focus and external focus in terms of nervous cost (21). Castaneda \& Gray skilled baseball players obtain better results from external focus, while the rookies get use of both external and internal focus of attention (2). De Bruin et al. observed no significant difference between internal and external focus in balance task (3). The common feature of these studies was the use of nonrhythmic motions and paying attention to the result of performance (outcome of performance) for measuring it. In other words, rhythmic motions or bimanual coordination motions have been less used in studying the effects of attentional instructions. 
Two main approaches including representation and non-representation (dynamic systems) have been considered by researchers of motor behavior. So far, studies on effects of type of attention on learning of motor activities have been mostly based on representation of information (16). But the theory of dynamic systems takes into account the variability in the internal production of motion in a more complex manner. Nonlinear perspective of dynamic systems provides unique descriptive aspects in the study of rhythmic motions. In this perspective, control parameters lead the behaviors of complex system. These parameters are factors that cause a system changes from a behavioral pattern another one. For instance, transition from one model to another one is observed as the rhythm of motion increases (29). Order parameters are variables that accurately describe the system behavior and follow predictable patterns (17). Relative phase in rhythmic motions is an efficient scale for describing the spatial-temporal relationship between two parts and is used as an order statistic in studying coordinated motions. There are two superior coordination patterns named inphase (Zero) and Anti-phase (180) that are known "Attractor" patterns. This means that if no certain phase difference is specified for coordination motions, motion pattern will tend to move towards one of the attractor patterns. This feature of coordinated motions is called "self-organization" (10). Other phasic patterns are out-of-phase or non-attractor. In other words, the control system is not willing to stay on these phase patterns. The biggest problem with the production of new patterns, compared with in-phase and anti-phase, is the relief from the reference patterns. Hodges \& Lee (1999) argued that power of inner tendencies is an obstacle to ease of access to other relative phases and showed that when two moving organs are driven to the relative phase other than in-phase and anti-phase, they immediately tend to return to these two patterns (7). However, they claimed that the time to return to the attractor patterns can be prolonged by feedback (5). The results many studies conducted on rhythmic motions suggest that the use of behavioral data (e.g. audio or visual signs) reduces the variability of motion and slows down the start of phase transition when the rhythm is increased. In addition, it is also effective in stability of motion patterns that are internally unstable $(9,12)$. Zanone \& Kelso (1997) showed that when the required coordination patterns match with internal coordination tendencies, the produced patterns would have the least error and variability and there is no need for alternative sources of support (29). Few studies conducted on the effects of attentional instruction on rhythmic motions have obtained the similar results as those studied these effects on nonrhythmic motions and most of them showed that external attention is better than internal attention in performing the task of coordination. For example, Lee, Blandin \& Proteau (1996) in the task of learning different relative phases and the effect of feedback as the attentional instruction, Hodges \& Lee (1999) in the task of drawing the relative phase on screen, Wanderoth et al. (2002) in the task of performing different relative phases in the presence of visual information and additional information, and James (2006) in the task of elbow flexion and extension in different relative phases showed these effects $(8,11$, 22). However, Zareian et al. (2011) found no significant difference between the effects of internal and external attentional instructions on Zero relative phase and 180 relative phase (30). According to constrained action hypothesis, they claimed that high and automatic internal tendencies make individuals needless to compliance with attentional instructions.

Since it is very important to maintain the relative phase when the rhythm is increased in performing many coordination patterns like sleight of hand gestures or playing the piano, this control parameter seems to be of effective factors in learning coordination patterns (James, 2006). The results of studies on this issue are not consistent; studies of Haken \& Kelso and Bunz (1985), Scholz \& Kelso (1990), and Kelso (1997) showed that increased rhythm of motion leads to the tendency of anti-phase pattern to in-phase pattern, or other out-of-phase patterns tend to go towards these two patterns $(6,10,17)$. Zero relative phase is more attractive than 180 relative phase. In other words, increased rhythm of motion increases the accuracy and stability of inphase patterns but decreases the stability of antiphase patterns. However, James (2006) and Zareian et al. (2011) showed that anti-phase pattern did not tend to go towards in-phase pattern when the rhythm increased and increased rhythm led to phasic stability in that pattern $(8,30)$. 
Given the conflicting results in relation to increased rhythms, the present paper aimed to study the effect of increasing the rhythm and attentional instruction on stability of relative phase of out-ofphase patterns [40, 90, and 139]. In other words, since non-attractor patterns have tendency to attractor patterns and increased rhythm accelerates this process, the main question of this study is that whether attentional instruction can increase the time of maintaining these pattern or not?

\section{MATERIAL \& METHOD}

Participant included the students of Physical Education in Allameh Tabataba'i University in the second semester of academic year 2013-2014. The sample consisted of 16 male and female students who voluntarily participated in this study. Their age ranged between 21 and $26(24.27 \pm 2.27)$. These people participated in the test session without previous experience and familiarity with the task and the plotter device. Before starting the test, medical information and records of participants were investigated using a questionnaire in order to ensure that they do not suffer from hearing and neurological disorders. Also, given that all the participants in this study should necessarily be right-handed, they were asked to fill out Briggs-Nebes Inventory (1975).

The device of processing and plotting bimanual coordination motions was used for performing experimental manipulations and measuring the variables. This device includes three mechanical, hardware, and software parts. The mechanical part consists of two levers that are placed on two bases. These bases move like a rail on a platform and are adjustable depending to the distance between the hands of subjects. The levers are able to simulate different bimanual coordination patterns between zero and 180 degree. The sampling rate in this research was $100 \mathrm{~Hz}$. In order to determine the validity and reliability of measurement tool, concurrent determination of validity method and retest at various times by Pearson correlation coefficient were used, respectively. Reliability and validity of the device were obtained, respectively, 0.84 and 0.9. Software of this device was equipped with a metronome that was adjustable to 60-240 BPM or 0.5-4 $\mathrm{Hz}$ with accuracy of $0.1 \mathrm{~Hz}$.
To calculate the relative phase, amplitude, and the distance of fluctuations center from their origin, Fourier series approximation was used. For this purpose, a single diagram was drawn from the diagrams related to two levers and sinus equations were used to calculate relative phase, amplitude, and coordination pattern. Descriptive data analysis and analysis of variance with repeated measures were also used to analyze the data. The subjects were randomly divided into experimental groups. Experimental plan was intragroup and intra-subject. Internal attention and external attention were the independent variables and increased rhythm was considered as the dependent (confounding) variable which was analyzed by 3 (attention) $\times 3$ (relative phase) $\times 4$ (frequency) analysis of variance with repeated measures. Mauchly test of sphericity was used to examine the homogeneity of variances and in groups where Mauchly test was significant, the scores of Greenhouse-Geisser test were used. In groups where the mutual effect was significant, Tukey and t-test with correction of degree of freedom by Bonferroni method were used to identify the couples that their difference was significant.

\section{Procedure}

Stages of data collection and conducting the experiment were done at Allameh Tabataba'i University of Tehran. Before entering the main stage of experiment, a preliminary pilot study was conducted in order to assess the tools and procedure of implementation and also to achieve a proper protocol. In order to consider the ethical and human codes, the participants were provided with information on the objective of study, methodology, and so on before the experiment. After coordination with the participants about the date, time and location of test, the subjects were asked to avoid any vigorous physical activities (particularly grueling exercises that stimulate the involvement of hands) before the experiment, have adequate sleep and nutrition, and avoid taking the drugs that affect their alertness and focus of attention. The subjects in this study performed the relative phases of 45, 90, and $135 \mathrm{deg}$. First of all, the subject sat down in a chair in front of the device and listened to the materials presented by the proctor. Then, the subject was asked to take the levers, adjust them to fit his/her hand, and try to maintain the defined phase for 20 seconds. The sound 
of a metronome stimulated the start of the task. This metronome was adjusted in a way that changed the phase every 5 seconds (from 60 BPM to 120, 180, and 240). The subjects were supposed to change their speed with increasing rhythm of metronome, while they were not aware that the rhythm was increasing every 5 seconds. In the first day of experiment, the task was performed without attentional instruction. In order to control the precedency and regency effect, half of the subjects ( 8 persons) performed the task at the second day under external attention, others performed that task at the first day under internal attention, and each group followed the opposite procedure at the third day. In external attentional instruction, the subjects should pay attention to the signs installed on the device and try to maintain the spatial position of motion levers consistent with the increased rhythm of metronome. Three 20-second attempts were determined for each phase. Phases were performed every other. In internal attentional instruction, colorful etiquettes were attached on arm and forearm of the subjects and they were asked to maintain the angle of their elbow according to the signs on the etiquettes. The signs on the device and the monitor were removed in this instruction. At the end of each attempt, the diagram of motion for each subject and a reference diagram were prepared. Before entering the main stage, two opportunities called warm-up were given to the subjects. The reason for selection of these relative phases was that they have the same difference (45 degree) with each other and also had the same distance with attractor phases.

\section{RESULTS}

In all three relative phases, the results of variance analysis showed that the effect of external attentional instruction on the stability of relative phase is more than other variables $(\mathrm{p}=0.001)$. In addition, no significant difference was observed between external attention and lack of using the attentional instruction in two relative phases of 45 and 139 degree. Generally, stability of relative phase in 90-degree relative phase was more than others.

Increased frequency reduced the relative phase stability in all phases. As shown in Figure 2, in all attentional instructions, relative stability of phases reduced with the increase in motion speed. However, external attentional instruction showed higher resistance to increased rhythm acted effective in maintaining the order parameters.

Table 1. Analysis of variance with repeated measures on each phase and comparison of attentional instructions.

\begin{tabular}{|c|c|c|c|c|}
\hline Relative Phase & $\eta^{2} \mathrm{p}$ & sig & $\mathrm{F}$ & $\mathrm{df}$ \\
\hline \multirow{5}{*}{45} & 0.52 & 0.001 & 16.28 & 2 \\
\hline & \multicolumn{4}{|c|}{ Pairwise Comparison } \\
\hline & $\mathrm{p}$ & MD (I-J) & $\mathrm{J}$ & I \\
\hline & 0.44 & 0.513 & internal & No-attention \\
\hline & & 0.001 & -2.28 & external \\
\hline \multirow{6}{*}{90} & $\eta^{2} \mathrm{p}$ & sig & $\mathrm{F}$ & $\mathrm{df}$ \\
\hline & 0.95 & 0.001 & 293.77 & 2 \\
\hline & \multicolumn{4}{|c|}{ Pairwise Comparison } \\
\hline & $\mathrm{p}$ & MD (I-J) & $\mathrm{J}$ & I \\
\hline & 0.001 & -1.47 & internal & No-attention \\
\hline & & 0.001 & -3.68 & external \\
\hline \multirow{6}{*}{135} & $\eta^{2} \mathrm{p}$ & sig & $\mathrm{F}$ & $\mathrm{df}$ \\
\hline & 0.92 & 0.001 & 176.49 & 2 \\
\hline & \multicolumn{4}{|c|}{ Pairwise Comparison } \\
\hline & $\mathrm{p}$ & $\mathrm{MD}(\mathrm{I}-\mathrm{J})$ & $\mathrm{J}$ & $\mathrm{I}$ \\
\hline & 0.790 & 0.31 & internal & No-attention \\
\hline & & 0.001 & -1.85 & external \\
\hline
\end{tabular}

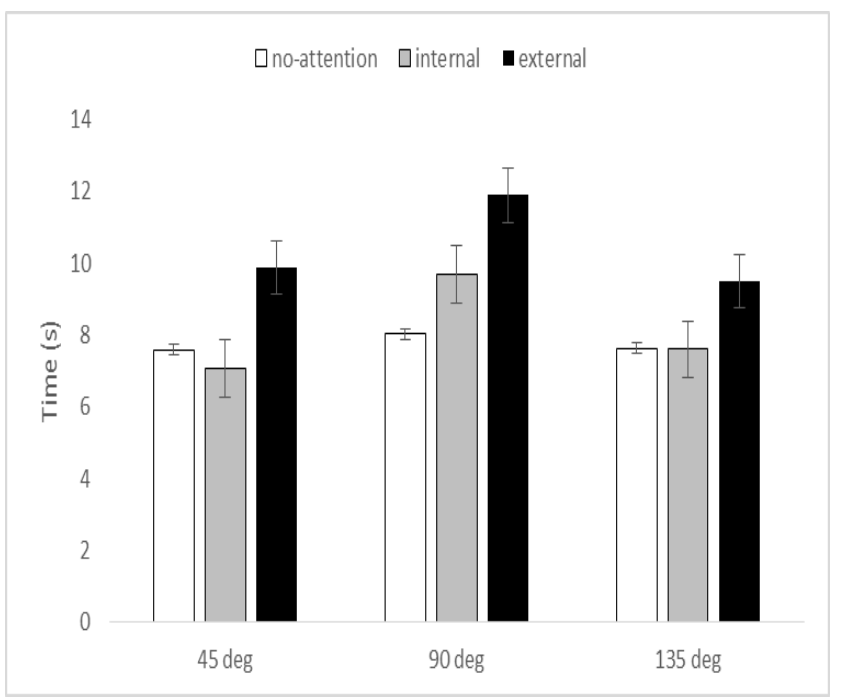

Figure 1. Effect of instructions on stability of different relative phases.

\section{DISCUSSION}

The results of this study showed that external attentional instruction lead to the highest stability in the corresponding relative phases. This confirms the results of previous studies on non-rhythmic motions conducted by Wulf \& Prinz (2001) and Wulf (2007) 
and is also consistent with constrained action hypothesis. The results obtained from these phases are also consistent with the findings of James (2006) who had used rhythmic motions. However, the results were inconsistent with the findings obtained by Zareian et al. (2011) on in-phase and anti-phase relative phases.

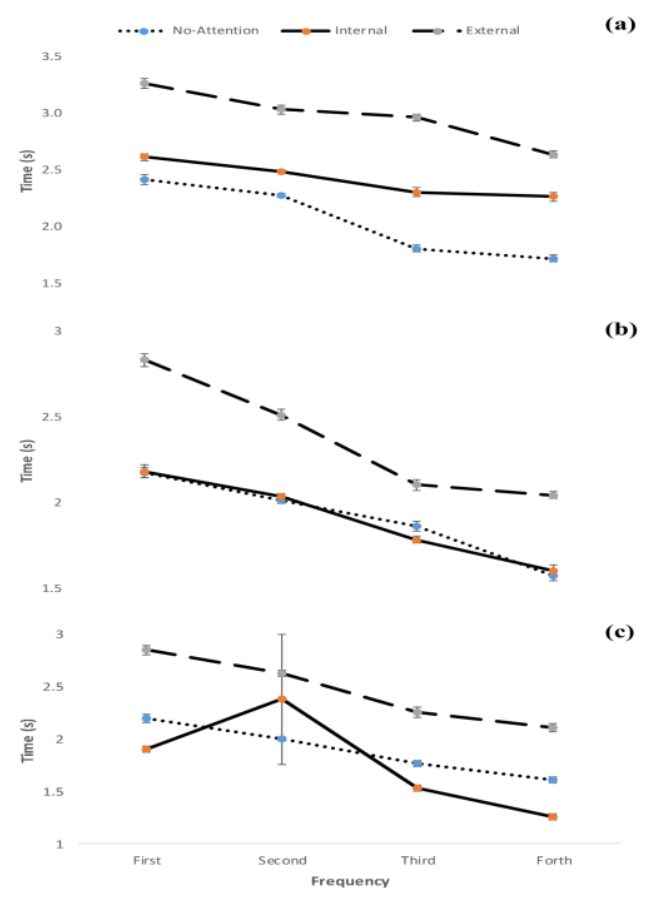

Figure 2. Stability of relative phases with increased rhythm (a) 90 degree (b) 135 degree (c) 45 degree.

The reason for obtaining conflicting results nonattractor phasic patterns can be attributed to complexity and difficulty of these phases. Zanone \& Kelso (28) claimed that when the coordinating pattern is consistent with internal coordination tendencies, the least error and variability would be seen in the produced patterns and there is no need for alternative sources of support. But in out-of-phase patterns, inconsistency of internal tendencies with phasic rhythmic tendencies highlights the need for alternative sources. Weak dynamism of internal tendencies of coordination system in out-of-phase patterns justifies the need for receiving supportive sources like attention and feedback. In fact, because of perturbation in such phases, a person can get use of external attentional instruction and increase the stability of the current phase. In other words, a person can apply external attention to resist more to speed control parameter changes and prevent the tendency to attractor patterns. The interesting thing about these three relative phases was that the total relative stability in 90 degree pattern was more than other phases which can be attributed to the spatial arrangement of patterns. In fact, familiarity of people with the relative phase and its ease of definition could of contributing factors. Another important point is that increased rhythm caused the tendency of all three relative phases to in-phase pattern and not anti-phase one. In other words, even in 135 degree relative phase which is closer to anti-phase pattern, increased rhythm did not induce tendency to this relative phase. More attractiveness of in-phase patterns is one of the reasons for this event. Ease of implementation, reducing the degrees of freedom, and compliance with internal tendencies are the factors that make this relative phase superior than others.

In general, it should be noted that external attentional instruction helps the relative stability of phase more than other attentional instructions. Additionally, no significant difference is observed between external attention and not using an attentional instruction in some of these relative phases. Hence, further studies are needed to investigate the effect of type of attentional instructions on bimanual rhythmic patterns and its effects in interaction with increased rhythm. In addition, studying other relative phases and the use of nonattractor relative phases in other tasks can broad the knowledge in this field.

\section{ACKNOWLEDGEMENT}

The author would like to thanks motor behavior students for help with researching this paper.

\section{REFRENCES}

1. Briggs G, Nebes RD. Patterns of hand preference in a student population. Cortex, 1975; 11: 230-238.

2. Castaneda B, Gray R. Effects of focus of attention on baseball batting performance in players of differing skill levels. Journal of Sport and Exercise Psychology, 2007; 29(1): 60.

3. De Bruin ED, Swanenburg J, Betschon E, Murer K. A randomized controlled trial investigating motor skill training as 
a function of attentional focus in old age. BMC Geriatrics, 2009; 9(1): 15.

4. Emanuel, Michal, Tal Jarus, and Orit Bart. Effect of focus of attention and age on motor acquisition, retention, and transfer: a randomized trial. Physical Therapy, 2008; 88(2): 251-260.

5. Fontaine RJ, Lee TD, Swinnen SP. Learning a new bimanual coordination pattern: Reciprocal influences of intrinsic and tobe-learned patterns. Canadian Journal of Experimental Psychology/Revue Canadienne de Psychologie Expérimentale. 1997; 51(1): 1 .

6. Haken H, Kelso JS, Bunz H. A theoretical model of phase transitions in human hand movements. Biological Cybernetics, 1985; 51(5): 347-56.

7. Hodges NJ, Lee TD. The role of augmented information prior to learning a bimanual visual--motor coordination task: Do instructions of the movement pattern facilitate learning relative to discovery learning? British journal of Psychology. 1999; 90(3): 389-403.

8. James, EG. Effects of Internal and External Focus of Attention on Relative Phase Stability in Bimanual Movements. A thesis presented to the faculty of Education university of Houston, May, 2006.

9. Kelso JA, Zanone PG. Coordination dynamics of learning and transfer across different effector systems. Journal of Experimental Psychology: Human Perception and Performance, 2002; 28(4): 776.

10. Kelso JS. Dynamic patterns: The self-organization of brain and behavior. MIT press; 1997.

11. Lee TD, Blandin Y, Proteau L. Effects of task instructions and oscillation frequency on bimanual coordination. Psychological Research, 1996; 59(2): 100-106.

12. Lohse KR, Wulf G, Lewthwaite R. Attentional focus affects movement efficiency. Skill acquisition in Sport: Research, Theory and Practice, 2012; 25: 40-58.

13. Maddox MD, Wulf G, Wright DL. The effect of an internal vs. external focus of attention on the learning of a tennis stroke. Journal of Exercise Psychology, 1999; 21(2): 78-85.

14. Perkins-Ceccato N, Passmore SR, Lee TD. Effects of focus of attention depend on golfers' skill. Journal of Sports Sciences, 2003; 21(8): 593-600.

15. Poolton JM, Maxwell JP, Masters RS, Van der Kamp J. Moving with an external focus: Automatic or simply less demanding. EJournal Bewegung und Training (E-Journal Movement and Training), 2007; 1: 43-44.

16. Schmidt, R.A. \& Lee, T.D. Motor control and learning: A behavior emphasis (5rd Ed). Champagin, IL: Human Kinetics. 2011.
17. Scholz JP, Kelso JA. Intentional switching between patterns of bimanual coordination depends on the intrinsic dynamics of the patterns. Journal of Motor Behavior, 1990; 22(1): 98-124.

18. Shana K. Carpenter, Keith R. Lohse, Alice F. Healy, Lyle E. Bourne Jr., Benjamin A. Clegg. External focus of attention improves performance in a speeded aiming task. Journal of Applied Research in Memory and Cognition, 2013; 2(1): 14-19.

19. Shea $\mathrm{CH}$, Wulf $\mathrm{G}$. Enhancing motor learning through externalfocus instructions and feedback. Human Movement Science, 1999; 18(4): 553-571.

20. Thomas, J. R., \& Nelson, J, K. Research Method in Physical Activity, Second Edition, Champaign, Illinois. Human Kinetics Books. 1990.

21. Vance J, Wulf G, Töllner T, McNevin N, Mercer J. EMG activity as a function of the performer's focus of attention. Journal of Motor Behavior, 2004; 36(4): 450-459.

22. Wenderoth N, Bock O, Krohn R. Learning a new bimanual coordination pattern is influenced by existing attractors. Motor Control-Champaign. 2002; 6(2): 166-182.

23. Wulf G, Dufek JS, Lozano L, Pettigrew C. Increased jump height and reduced EMG activity with an external focus. Human Movement Science, 2010; 29(3): 440-448.

24. Wulf G, McConnel N, Gärtner M, Schwarz A. Enhancing the learning of sport skills through external-focus feedback. Journal of Motor Behavior, 2002; 34(2): 171-182.

25. Wulf G, Prinz W. Directing attention to movement effects enhances learning: A review. Psychonomic Bulletin \& Review, 2001; 8(4): 648-660.

26. Wulf, G. Attention and motor skill learning. Champaign, IL: Human Kinetics, 2007; 89-94.

27. Zachry T, Wulf G, Mercer J, Bezodis N. Increased movement accuracy and reduced EMG activity as the result of adopting an external focus of attention. Brain Research Bulletin. 2005; 67(4): 304-309.

28. Zanone PG, Kelso JA. Evolution of behavioral attractors with learning: non-equilibrium phase transitions. Journal of Experimental Psychology: Human Perception and Performance, 1992; 18(2): 403.

29. Zanone PG, Kelso JS. Coordination dynamics of learning and transfer: collective and component levels. Journal of Experimental Psychology: Human Perception and Performance, 1997; 23(5): 1454.

30. Zareian, E, Bahram. A., \& Sohrabi, M. Effect of type of attentional instruction and increasing frequency on relative phase stability of coordination bimanual movements. Research in sport management and motor Behavior, 2011; 1(1): 1-11. 